\title{
EXPONENTIAL ACTIONS, ORBITS AND THEIR KERNELS
}

\author{
J. Ludwig and C. Molitor-Braun
}

Let $\mathfrak{g}$ be a nilpotent Lie algebra which is an exponential $\mathfrak{a}$-module, $\mathfrak{a}$ being an exponential algebra of derivations of $\mathfrak{g}$. Put $\mathfrak{A}=\exp \mathfrak{a}$ and $\mathfrak{G}=\exp \mathfrak{g}$. If $\Omega$ is a closed orbit of $\mathfrak{g}^{*}$ under the action of $\mathfrak{A}$, then $\operatorname{Ker} \Omega \cap \mathcal{S}(\mathfrak{G})$ is dense in Ker $\Omega$ for the topology of $L^{1}(\mathfrak{G})$ and the algebra $\operatorname{Ker} \Omega / \widetilde{\jmath}(\Omega)$ is nilpotent, where $\tilde{\jmath}(\Omega)$ denotes the minimal closed ideal of $L^{1}(\mathfrak{G})$ whose hull is $\Omega$. Moreover, the $\mathfrak{A}$-prime ideals of $L^{1}(\mathfrak{B})$ coincide with the kernels $\operatorname{Ker} \Omega$, where $\Omega$ denotes an arbitrary orbit (not necessarily closed) in $\mathfrak{g}^{*}$.

\section{INTRODUCTION}

In abstract harmonic analysis a lot of efforts has been made to generalise certain properties of the group algebra $L^{1}(\mathbb{R})$ (like the Wiener property, questions of spectral synthesis, the characterisation of prime ideals) to nonabelian groups. For nilpotent Lie groups these questions have among others been solved by Leptin (Wiener property in $\left.L^{1}(\mathfrak{B}),[10]\right)$ and by Ludwig ([15] for the Wiener property in the Schwartz algebra $\mathcal{S}(\mathfrak{G})$ and [14] for the characterisation of prime ideals in $\mathcal{S}(\mathfrak{B})$ and $L^{1}(\mathfrak{G})$ ). Some partial results are known for solvable exponential groups [13], but a lot of questions remain open. In this paper we are therefore going to introduce an intermediate step between nilpotent and exponential Lie groups: the exponential actions on nilpotent Lie groups. Some special examples of exponential actions have already appeared in literature. In [20] Poguntke considers a connected, simply connected exponential Lie group $\mathfrak{B}=\exp \mathfrak{g}$ which acts in a natural way on $\mathfrak{N}=\exp \mathfrak{n}$, where $\mathfrak{n}$ is the nilradical of $\mathfrak{g}$. This induces an action on $L^{1}(\mathfrak{N})$ and $\mathcal{S}(\mathfrak{N})$. Poguntke uses this action in his description of the algebraically irreducible representations of an exponential Lie group. In [13] Ludwig uses particular exponential actions to study problems of symmetry and a special case of the Wiener property for exponential Lie groups. These two examples lead to the following definition of exponential actions on nilpotent Lie groups.

Definition: Let $\mathfrak{g}$ be a nilpotent Lie algebra. Let $\mathfrak{a}$ be an exponential solvable algebra of derivations of $\mathfrak{g}$, containing ad $\mathfrak{g}$. We define $\mathfrak{g}$ to be an exponential $\mathfrak{a}$-module

Received 11th December, 1997

Supported by the research grant MEN/CUL/95/05 at the Centre Universitaire de Luxembourg.

Copyright Clearance Centre, Inc. Serial-fee code: 0004-9729/98 \$A2.00+0.00. 
if, for every irreducible subquotient $\mathfrak{g}_{1} / \mathfrak{g}_{2}$, which is of dimension 1 or 2 as $\mathfrak{a}$ is solvable, the action of $\mathfrak{a}$ on a vector of the complexification

$$
\left(\mathfrak{g}_{1} / \mathfrak{g}_{2}\right)_{\mathbb{C}}=\left(\mathfrak{g}_{1} / \mathfrak{g}_{2}\right) \oplus i\left(\mathfrak{g}_{1} / \mathfrak{g}_{2}\right)
$$

is given by

$$
A(X)=\varphi(A)(1+i \omega) X \quad \bmod \left(\mathfrak{g}_{2}\right)_{\mathbb{C}}, \quad \text { for all } A \in \mathfrak{a}, X \in \mathfrak{g}_{1},
$$

with $\omega \in \mathbb{R}, \varphi \in \mathfrak{a}^{*}$. We then say that we have an exponential action of $\mathfrak{a}$ on $\mathfrak{g}$.

Let $\mathfrak{A}=\exp \mathfrak{a}$ and $\mathfrak{G}=\exp \mathfrak{g}$ be the simply connected, connected Lie groups associated with $\mathfrak{a}$ and $\mathfrak{g}$. The group $\mathfrak{A}$ is exponential solvable and defines the following actions: for every $a=\exp A \in \mathfrak{A}$,

$$
\begin{aligned}
a(X)=\sum_{k=0}^{\infty} \frac{1}{k !} A^{k}(X), & \text { for all } X \in \mathfrak{g} \\
{ }^{a} x={ }^{a}(\exp X)=\exp (a(X)), & \text { if } x=\exp X \in \mathfrak{G} \\
\left({ }^{a} \pi\right)(x)=\pi\left({ }^{-1} x\right), & \text { for all } \pi \in \widehat{\mathfrak{G}}, x \in \mathfrak{G} \\
f^{a}(x)=\delta(a) f\left({ }^{a} x\right), & \text { for all } f \in L^{1}(\mathfrak{G}) .
\end{aligned}
$$

Here $\delta$ stands for the modular function defined such that

$$
\int f^{a}(x) d x=\int f(x) d x, \text { for all } f \in L^{1}(\mathfrak{G}) .
$$

Moreover,

$$
\begin{aligned}
\left\langle a^{*} \cdot l, X\right\rangle & =\left\langle l, a^{-1}(X)\right\rangle, \\
\left\langle A^{*} \cdot l, X\right\rangle & =\langle l,-A(X)\rangle, \quad \text { for all } l \in \mathfrak{g}^{*}, X \in \mathfrak{g} .
\end{aligned}
$$

Of course, the Schwartz algebra $\mathcal{S}(\mathfrak{G})$ of rapidly decreasing $C^{\infty}$ functions on $\mathfrak{G}$ is invariant under the action of $\mathfrak{A}=\exp \mathfrak{a}[16]$.

EXAMPLES. (a) Let $\mathfrak{A}$ be the group of all $n \times n$ upper-triangular invertible real matrices with positive entries on the diagonal. Then $\mathfrak{A}$ acts exponentially on the Abelian group $\mathfrak{G}=\mathbb{R}^{n}$.

(b) The same group $\mathfrak{A}$ acts by conjugation on the nilpotent group of all $n \times n$ upper-triangular real matrices with 1 's on the diagonal.

By the Kirillov theory [9] we know that coadjoint orbits play an important role in the representation theory of nilpotent Lie groups. The same will be true in our situation, where orbits are defined as follows. 
Definition: For all $l \in \mathfrak{g}^{*}$, let $\Omega_{l}$ denote the orbit of $l$ given by

$$
\Omega_{l}=\left\{a^{*} \cdot l \mid a=\exp A \in \mathfrak{A}\right\} .
$$

Recall that every $\pi \in \widehat{\mathfrak{G}}$ is obtained in the following way: there exist $l \in \mathfrak{g}^{*}$ and a polarisation $\mathfrak{h} \subset \mathfrak{g}$ of $l$ such that, if $\chi_{l}(h)=e^{-i\langle l, \log h\rangle}$ is the character defined by $l$ on $\mathfrak{H}=\exp \mathfrak{h}$, then $\pi$ is equivalent to the induced representation $\operatorname{ind}_{\mathfrak{H}}^{\mathfrak{B}} \chi_{\mathfrak{l}}$. It is easy to check that for every $a \in \mathfrak{A}$, the representation ${ }^{a} \pi$ is unitarily equivalent to the induced representation ind $\exp _{\operatorname{Ba}(a \cdot b)}^{\mathfrak{B}} \chi_{a^{*} \cdot l}$. This fact justifies the following definition of the kernel of an orbit.

Definition: Let $\pi=\operatorname{ind}_{\mathfrak{H}}^{\mathfrak{E}} \chi_{l} \in \widehat{\mathfrak{G}}$. We define the kernel of the orbit $\Omega_{l}$ by

$$
\operatorname{Ker} \Omega_{l}=\left\{\left.f \in L^{1}(\mathfrak{G})\right|^{a} \pi(f)=0 \quad \forall a \in \mathfrak{A}\right\} .
$$

REMARKS.

(a) Orbits of exponential actions are not necessarily closed. For instance, let $\mathfrak{A}$ be the connected component of the affine group. Its Lie algebra $\mathfrak{a}$ is spanned by $\{X, Y\}$ where $[X, Y]=Y$. Take $\mathfrak{g}=[\mathfrak{a}, \mathfrak{a}]=\mathbb{R} Y$ and $l=Y^{*} \mid \mathfrak{g}$. Then the orbit of $l$ under the action of $\mathfrak{A}$ is $\mathbb{R}_{+}^{*}\left(Y^{*} \mid \mathfrak{g}\right)$ which is not closed.

(b) The distinction between closed and nonclosed orbits will play an important role in our future results.

(c) Every orbit of an exponential action is open in its closure. This is shown by Pukanszky [22, Corollary 1] and Conze $[1$, p.11].

In this paper we are going to prove the following facts about orbits of exponential actions on nilpotent Lie groups.

(a) If the orbit $\Omega$ is closed, then $\operatorname{Ker} \Omega \cap \mathcal{S}(\mathfrak{G})$ is dense in $\operatorname{Ker} \Omega$ for the topology of $L^{1}(\mathfrak{G})$ (Theorem 4.2). This result is important since properties for function algebras of nilpotent Lie groups are often first proved for the Schwartz algebra $\mathcal{S}(\mathfrak{G})$ and then transferred to $L^{1}(\mathfrak{G})$.

(b) Let $\Omega$ be a closed orbit. Then there exists a minimal closed ideal $\tilde{\jmath}(\Omega)$ in $L^{1}(\mathfrak{G})$ such that its hull satisfies $h(\tilde{\jmath}(\Omega))=\Omega$ and such that $\tilde{\jmath}(\Omega)$ is contained in every ideal $I$ of $L^{1}(\mathfrak{B})$ satisfying $h(I) \subset \Omega([15,3.2]$ and [17]). We prove that the algebra $\operatorname{Ker} \Omega / \widetilde{\jmath}(\Omega)$ is nilpotent, which means that there exists $M \in \mathbb{N}$ such that

$$
(\operatorname{Ker} \Omega / \widetilde{\jmath}(\Omega))^{M}=\{0\}
$$

(Theorem 5.1).This, by the way, is a generalised spectral synthesis result. 
(c) A proper $\mathfrak{A}$-invariant ideal $I$ of $L^{1}(\mathfrak{B})$ is said to be $\mathfrak{A}$-prime whenever for all $\mathfrak{A}$-invariant ideals $I_{1}$ and $I_{2}$ of $L^{1}(\mathfrak{G})$,

$$
I_{1} * I_{2} \subset I \Rightarrow I_{1} \subset I \text { or } I_{2} \subset I
$$

We prove that the proper closed $\mathfrak{A}$-prime ideals of $L^{1}(\mathfrak{G})$ coincide with the kernels $\operatorname{Ker} \Omega$ of orbits $\Omega$ of $\mathfrak{g}^{*}$ (Theorem 5.3).

The main tool to prove the preceding results is the use of functions of a generalised Schwartz space

$$
\mathcal{E S}\left(\mathbb{R}^{n}, \mathfrak{G} / \mathfrak{H} \times \mathfrak{G} / \mathfrak{H}, l\right) \equiv \mathcal{E S}\left(\mathbb{R}^{n}, \mathbb{R}^{k} \times \mathbb{R}^{k}\right)
$$

(see Section 3). The elements of this space can be identified with the kernels of operators of the form ${ }^{a} \pi(f)$ with $f \in \mathcal{S}(\mathfrak{G})$. These functions will allow us to show the essential relation between $\operatorname{Ker} \Omega$ and $\operatorname{Ker} \Omega \cap \mathcal{S}(\mathfrak{B})$ (Proposition 4.1).

\section{FunCtions WhOSE NONCOMMUTATIVE FOURIER tRANSFORMS HAVE COMPACT SUPPORTS}

For locally compact Abelian groups $\mathfrak{G}$ the functions $f \in L^{1}(\mathfrak{G})$ whose Fourier transforms have compact support, play an important role in the proofs of problems of spectral analysis and synthesis. In a generalised form the same remains true in our situation (Proposition 2.2, Theorem 4.2). But first we mention some results on stabilisers and orbits.

DEFINITION: For all $\pi=\operatorname{ind}_{\mathfrak{H}}^{\mathfrak{G}} \chi_{l} \in \widehat{\mathfrak{B}}$, we define the following stabilisers:

$$
\begin{aligned}
\mathfrak{a}_{l} & =\left\{A \in \mathfrak{a} \mid A^{*} \cdot l=0\right\} \\
\mathfrak{A}_{\pi} & =\left\{a \in \mathfrak{A} \mid{ }^{a} \pi \text { and } \pi \text { are unitarily equivalent }\right\} \\
\mathfrak{a}_{\pi} & =\left\{A \in \mathfrak{a} \mid \exp A \in \mathfrak{A}_{\pi}\right\}=\log \mathfrak{A}_{\pi}
\end{aligned}
$$

REMARKS.

(a) As the algebra $\mathfrak{a}$ is exponential and acts exponentially on $\mathfrak{g}^{*}$, we know that the stabiliser

$$
\mathfrak{A}_{l}=\left\{a \in \mathfrak{A} \mid a^{*} \cdot l=l\right\}
$$

is the exponential of $\mathfrak{a}_{l}[1,3.5]$.

(b) One shows that $\mathfrak{A}_{\pi}=$ Ad $\mathfrak{G} \cdot \mathfrak{A}_{l}$ and hence that $\mathfrak{A}_{\pi}$ is a connected subgroup of $\mathfrak{A}[\mathbf{1 8}, 2.9]$. 
Proposition 2.1. The spaces $\mathfrak{A} / \mathfrak{A}_{\pi}$ and $\Omega_{l} / \mathrm{Ad}^{*} \mathfrak{G}$ (identified with a subspace of $\widehat{\mathfrak{B}}$ ) are homeomorphic.

Proof: The mapping

$$
\begin{aligned}
\varphi: \mathfrak{A} & \rightarrow \Omega_{l} \\
a & \mapsto a^{*} \cdot l
\end{aligned}
$$

is a continuous, open surjection [6]. The same is true for the projection

$$
p: \Omega_{l} \rightarrow \Omega_{l} / \operatorname{Ad}^{*} \mathfrak{G}
$$

for the mapping

$$
\varphi_{1}=p \circ \varphi: \mathfrak{A} \rightarrow \Omega_{l} / \mathrm{Ad}^{*} \mathfrak{G}
$$

and for the projection

$$
p_{1}: \mathfrak{A} \rightarrow \mathfrak{A} / \mathfrak{A}_{\pi} .
$$

Since $\mathfrak{A}_{\pi}$ is the stabiliser of $\Omega_{l}=\operatorname{Ad}^{*} \mathfrak{G} \cdot l$, there exists a unique bijection $\phi$ of $\mathfrak{A} / \mathfrak{A}_{\pi}$ onto $\Omega_{l} / \mathrm{Ad}^{*} \mathfrak{G}$ such that $\phi \circ p_{1}=\varphi_{1}$. In fact $\phi$ is a homeomorphism.

Definition: For every $f \in L^{1}(\mathfrak{B})$, we define supp $\widehat{f}$ by

$$
\operatorname{supp} \hat{f}=\overline{\{\pi \in \widehat{\mathfrak{B}} \mid \pi(f) \neq 0\}} \widehat{\widehat{B}} .
$$

PROPOSITION 2.2. There exists an approximate identity $\left(g_{\nu}\right)_{\nu}$ in $\mathcal{S}(\mathfrak{G})$ such that supp $\widehat{g_{\nu}}$ is compact for all $\nu$.

PRoOF: Let $\left(V_{j}\right)_{j \in \mathbb{N}}$ be a basis of neighbourhoods of the identity element $e$ of $\mathfrak{G}$. For each $j \in \mathbb{N}$ there exists a compact neighbourhood $K_{j}$ of $e$ in $\mathfrak{G}$ such that $\left(K_{j}\right)^{j} \subset V_{j}$. Of course the $K_{j}$ 's also form a basis of compact neighbourhoods of $e$, which we may even choose decreasing. We then construct a family of real positive functions $f_{j} \in \mathcal{S}(\mathfrak{B})$ such that $\int f_{j}(x) d x=1$ and supp $f_{j} \subset K_{j}$ for all $\mathrm{j}$. Hence $\operatorname{supp}\left(f_{j}\right)^{* j} \subset\left(K_{j}\right)^{j} \subset V_{j}, \lim _{j \rightarrow+\infty}\left(f_{j}\right)^{* j} * g=g$ and even $\lim _{(j, n) \rightarrow \infty}\left(f_{j}\right)^{* n} * g=g$ for all $g \in \mathcal{S}(\mathfrak{G})$, where $(j, n) \rightarrow \infty$ means that $j \rightarrow+\infty, n \rightarrow+\infty$ and $j \geqslant n$ [16]. By [4] we may now choose for each $n \in \mathbb{N}, \varphi_{n} \in C^{\infty}(\mathbb{R}), \varepsilon_{n}>0$ and $C_{n}$ such that $\varphi_{n} \equiv 0$ in a small neighbourhood of $0, \sup _{t \in C_{n}}\left|\left(\varphi_{n}(t)-t^{n}\right)^{(r)}\right|<\varepsilon_{n}$ for $r=0,1, \ldots,(n-1)$, $\varepsilon_{n} \rightarrow 0$ and $\varphi_{n}(t)=t^{n}$ for $t \notin C_{n}$, where $C_{n}$ is a compact neighbourhood of 0 in $\mathbb{R}$ contained in $[-1,1]$. The functional calculus of Dixmier [4] and Hulanicki [8] shows that $\varphi_{n}\{f\} * g-f^{n} * g$ tends to 0 in $\mathcal{S}(\mathfrak{B})$ if $n \rightarrow+\infty$, independently of $f$, if $f$ is selfadjoint with support in a fixed compact set and if $\|f\|_{1} \leqslant 1$ (see the proof of 
Theorem 2.1 in [8]). Hence $\varphi_{n}\left\{f_{j}\right\} * g$ converges to $g$ in $\mathcal{S}(\mathfrak{B})$. Let now $\varepsilon>0$ be such that $\varphi_{n} \equiv 0$ on $[-\varepsilon, \varepsilon]$. As there exists a compact set $C$ in $\widehat{\mathfrak{G}}$ such that $\left\|\pi\left(f_{j}\right)\right\|_{\text {op }}<\varepsilon$ for every $\pi \notin C[5], \pi\left(\varphi_{n}\left\{f_{j}\right\}\right)=\varphi_{n}\left(\pi\left(f_{j}\right)\right)=0$ for every $\pi \notin C$. The $\varphi_{n}\left\{f_{j}\right\}$ 's may then be taken as the approximate identity $\left(g_{\nu}\right)_{\nu}$.

If the $\mathfrak{A}$-orbit $\Omega_{l}$ corresponding to $\pi=\operatorname{ind}_{\mathfrak{\zeta}}^{\mathfrak{G}} \chi_{l}$ is closed, $\Omega_{l} / \mathrm{Ad}^{*} \mathfrak{G}$ is a closed subspace of $\mathfrak{g}^{*} / \mathrm{Ad}^{*} \mathfrak{G}$ (identified with $\widehat{\mathfrak{G}}$ ). If moreover supp $\widehat{f}$ is compact, then $\overline{\left\{a \in \mathfrak{A} / \mathfrak{A}_{\pi} \mid{ }^{a} \pi(f) \neq 0\right\}}$ is a closed compact subset of $\mathfrak{A} / \mathfrak{A}_{\pi}$, which can be identified with a closed compact subset of $\operatorname{supp} \hat{f} \cap\left(\Omega_{l} / \operatorname{Ad}^{*} \mathfrak{G}\right)$, according to Proposition 2.1. This is in particular true for

$$
\overline{\left\{a \in \mathfrak{A} / \mathfrak{A}_{\pi} \mid{ }^{a} \pi\left(g_{\nu}\right) \neq 0\right\}},
$$

where $\left(g_{\nu}\right)_{\nu}$ is the approximate identity of Proposition 2.2.

\section{KERNELS OF ${ }^{a} \pi(f)$ OPERATORS}

For every Schwartz function $f$ on the nilpotent Lie group $\mathfrak{G}$, the operator $\pi(f)$ is a kernel operator. Its kernel is given by a Schwartz function in two variables that satisfies a certain covariance condition. These kernel functions have to be generalised to take into account the exponential action on $\widehat{\mathfrak{G}}$ : if $f \in \mathcal{S}(\mathfrak{B})$ is fixed, the kernel of the operator ${ }^{a} \pi(f)=\pi\left(f^{a}\right)$ is a function of $a$, denoted $F(a ; x ; y)$ that has to decrease exponentially in some directions of $a$. In order to be more precise, we have to use the concept of a coexponential basis.

Let $\mathfrak{B}=\exp \mathfrak{b}$ be an exponential solvable Lie group and $\mathfrak{P}=\exp \mathfrak{p}$ a closed connected, simply connected subgroup of $\mathfrak{B}$. Then there exist a basis $\widetilde{\mathcal{B}}=\left\{b_{1}, \ldots, b_{p}\right\}$ of $\mathfrak{b}$ and an integer $n \leqslant p$ such that $\mathcal{B}=\left\{b_{1}, \ldots, b_{n}\right\}$ defines a basis of $\mathfrak{b} / \mathfrak{p}, \mathcal{B}_{1}=$ $\left\{b_{n+1}, \ldots, b_{p}\right\}$ is a basis of $\mathfrak{p}$ and such that the mapping

$$
\begin{aligned}
& \widetilde{E}_{\widetilde{\mathcal{B}}}: \mathbb{R}^{p} \longrightarrow \mathfrak{B} \\
& \widetilde{E}_{\widetilde{\mathcal{B}}}\left(t_{1}, \ldots, t_{p}\right)=\exp t_{1} b_{1} \ldots \exp t_{p} b_{p}
\end{aligned}
$$

is a diffeomorphism $[\mathbf{1}, \mathbf{2 0}]$. Let $\mathfrak{V} \subset \mathfrak{B}$ be defined by

$$
\mathfrak{V}=\widetilde{E}_{\widetilde{\mathcal{B}}}\left(\mathbb{R}^{n} \times(0, \ldots, 0)\right),
$$

with $(0, \ldots, 0) \in \mathbb{R}^{n-p}$. Obviously the subset $\mathfrak{V}$ is closed in $\mathfrak{B}$ and the equality $\mathfrak{B}=\mathfrak{V} \cdot \mathfrak{P}$ gives us a diffeomorphism between $\mathfrak{B}$ and $\mathfrak{V} \times \mathfrak{P}$. Moreover the mapping

$$
\begin{aligned}
& E_{\mathcal{B}}: \mathbb{R}^{n} \longrightarrow \mathfrak{V} \\
& E_{\mathcal{B}}\left(t_{1}, \ldots, t_{n}\right)=\widetilde{E}_{\widetilde{\mathcal{B}}}\left(t_{1}, \ldots, t_{n}, 0, \ldots, 0\right)
\end{aligned}
$$


where $(0, \ldots, 0) \in \mathbb{R}^{n-p}$ is a diffeomorphism between $\mathbb{R}^{n}$ and $\mathfrak{V}$.

Definition: The basis $\mathcal{B}=\left\{b_{1}, \ldots, b_{n}\right\}$ of $\mathfrak{V}$ is called a coexponential basis of $\mathfrak{b}$ with respect to $\mathfrak{p}$.

Let us now apply the preceding construction to $\mathfrak{A}$ and $\mathfrak{A}_{\pi}: \mathcal{B}, \widetilde{\mathcal{B}}$ and $\mathfrak{V}$ will be defined as previously, but for the groups $\mathfrak{A}$ and $\mathfrak{A}_{\pi}$ instead of $\mathfrak{B}$ and $\mathfrak{P}$. The coexponential basis of $\mathfrak{a}$ with respect to $\mathfrak{a}_{\pi}$ defines a homeomorphism between $\mathfrak{A} / \mathfrak{A}_{\pi}$ (and $\Omega_{l} / \operatorname{Ad}^{*} \mathfrak{G}$, by Proposition 2.1) and $\mathbb{R}^{n}$. In particular, $\Omega_{l} / \operatorname{Ad}^{*} \mathfrak{G}$ is a locally compact Hausdorff subspace of $\mathfrak{g}^{*} / \operatorname{Ad}^{*} \mathfrak{G}$. Moreover, the mapping $E_{\mathfrak{B}}$ allows us to identify $\mathfrak{V}$ with $\mathbb{R}^{n}$. Hence, for every $\alpha \in \mathbb{R}^{n}$ and every $a \in \mathfrak{V},\langle\alpha, a\rangle$ will denote the number $\left\langle\alpha, E_{\mathcal{B}}^{-1}(a)\right\rangle$, obtained by the scalar product in $\mathbb{R}^{n}$. We then define the generalised Schwartz spaces of kernel functions.

Definition: (a) We note by $\mathcal{E S}\left(\mathfrak{V}, \mathbb{R}^{k} \times \mathbb{R}^{k}\right)$ for the space of all complex valued smooth functions on $\mathfrak{V} \times \mathbb{R}^{k} \times \mathbb{R}^{k}$ such that

$$
\left(a ; s_{1}, \ldots, s_{k} ; t_{1}, \ldots, t_{k}\right) \mapsto e^{\langle\alpha, a\rangle} F\left(a ; s_{1}, \ldots, s_{k} ; t_{1}, \ldots, t_{k}\right)
$$

is a Schwartz function for all $\alpha \in \mathbb{R}^{n}$.

(b) We write $\mathcal{E S}(\mathfrak{V}, \mathfrak{G} / \mathfrak{H} \times \mathfrak{G} / \mathfrak{H}, l)$ for the space of all complex valued smooth functions on $\mathfrak{V} \times \mathfrak{G} \times \mathfrak{G}$ such that:

(i) $F\left(a ; x h ; x^{\prime} h^{\prime}\right)=\overline{\chi_{l}(h)} \chi_{l}\left(h^{\prime}\right) F\left(a ; x ; x^{\prime}\right) \quad$ for all $x, x^{\prime} \in \mathfrak{G}, h, h^{\prime} \in \mathfrak{H}$

(ii) for every coexponential basis $\left\{c_{1}, \ldots, c_{k}\right\}$ in $\mathfrak{g}$ with respect to $\mathfrak{h}$ and every $\alpha$ in $\mathbb{R}^{n}$, the function

$$
\begin{aligned}
& \left(a ; s_{1}, \ldots, s_{k} ; t_{1}, \ldots, t_{k}\right) \mapsto \\
& \quad e^{\langle\alpha, a)} F\left(a ; \exp s_{1} c_{1} \cdots \exp s_{k} c_{k} ; \exp t_{1} c_{1} \cdots \exp t_{k} c_{k}\right)
\end{aligned}
$$

is a Schwartz function. For a fixed coexponential basis of $g$ with respect to $\mathfrak{h}$ we may of course identify the spaces $\mathcal{E S}\left(\mathfrak{V}, \mathbb{R}^{k} \times \mathbb{R}^{k}\right)$ and $\mathcal{E S}(\mathfrak{V}, \mathfrak{G} / \mathfrak{H} \times \mathfrak{G} / \mathfrak{H}, l)$. In particular, if $\mathfrak{V}=0$ we have the space $\mathcal{S}\left(\mathbb{R}^{k} \times \mathbb{R}^{k}\right) \equiv \mathcal{S}(\mathfrak{G} / \mathfrak{H} \times \mathfrak{G} / \mathfrak{H}, l)$. For details, see $[17,18]$. Similar definitions may be found in [11].

The kernel functions are obtained as follows: if $f \in \mathcal{S}(\mathfrak{G})$ and $\pi=\operatorname{ind}_{\mathfrak{H}}^{\mathfrak{B}} \chi_{l} \in \widehat{\mathfrak{B}}$, the operator $\pi(f)$ has a kernel $f_{\pi} \in \mathcal{S}(\mathfrak{G} / \mathfrak{H} \times \mathfrak{G} / \mathfrak{H}, l)$ given by

$$
f_{\pi}(x, y)=\int_{\mathfrak{H}} f\left(x h y^{-1}\right) \chi_{l}(h) d h, \quad \text { for all } x, y \in \mathfrak{G} .
$$

Moreover the mapping

$$
\begin{aligned}
\mathcal{S}(\mathfrak{G}) & \rightarrow \mathcal{S}(\mathfrak{G} / \mathfrak{H} \times \mathfrak{G} / \mathfrak{H}, l) \\
f & \mapsto f_{\pi}
\end{aligned}
$$


is continuous [7]. Hence the relationship ${ }^{a} \pi(f)=\pi\left(f^{a}\right)$ shows that the operators ${ }^{a} \pi(f)$ have a kernel function that depends continuously on $a$. Let us write $F(a ; . ;$. for the kernel function of ${ }^{a} \pi(f)$ if $a \in \mathfrak{V}$ and $\widetilde{F}(a ; . ;$.$) for the kernel function of the$ same operator if we take $a \in \mathfrak{A}$. For our future arguments the choice of the preceding coexponential bases is not arbitrary, but has to be taken in accordance with the following result.

Theorem 3.1. Let $l \in \mathfrak{g}^{*}$. Then there exist a Pukanszky polarisation $\mathfrak{h}$ of $l$ in $\mathfrak{g}$, a coexponential basis in $\mathfrak{g}$ with respect to $\mathfrak{h}$ and a coexponential basis in $\mathfrak{a}$ with respect to $a_{\pi}$ for which there is a continous mapping

$$
\begin{aligned}
\mathcal{E S}(\mathfrak{V}, \mathfrak{G} / \mathfrak{H} \times \mathfrak{G} / \mathfrak{H}, l) & \rightarrow \mathcal{S}(\mathfrak{G}) \\
F & \mapsto f
\end{aligned}
$$

such that ${ }^{a} \pi(f)$ is an operator with kernel $F(a ; . ;$.$) for all a \in \mathfrak{V}$. Moreover, in this basis the space $\mathcal{E S}(\mathfrak{V}, \mathfrak{G} / \mathfrak{H} \times \mathfrak{G} / \mathfrak{H}, l)$ is identified with the space $\mathcal{E} \mathcal{S}\left(\mathfrak{V}, \mathbb{R}^{k} \times \mathbb{R}^{k}\right)$.

Proof: See $[19,5.3]$.

Let $\mathfrak{V}$ and $\mathfrak{A}$ be equipped with their fixed bases and endowed with the Lebesgue measures on the corresponding $\mathbb{R}^{n}$ and $\mathbb{R}^{p}$ spaces. Since the relationship $\mathfrak{A}=\mathfrak{V} \cdot \mathfrak{A}_{\pi}$ gives a unique decomposition $u=v \cdot a, v \in \mathfrak{V}$ and $a \in \mathfrak{A}_{\pi}$, of every element $u$ of $\mathfrak{A}$, we may define the projection $p$ of $\mathfrak{A}$ onto $\mathfrak{V}$ by

$$
\begin{aligned}
p: \mathfrak{A} & \rightarrow \mathfrak{V} \\
u=v \cdot a & \mapsto p(u)=v .
\end{aligned}
$$

This projection is a continuous mapping of $\mathfrak{A}$ onto $\mathfrak{V}$.

For technical reasons we have to make some transformations: let $\psi \in C_{c}^{\infty}(\mathfrak{A})$ with compact support $K_{0} \subset \mathfrak{A}$ such that

$$
\psi \geqslant 0, \quad \psi(0)>0, \quad \int_{\mathfrak{A}} \psi(a) d a=1
$$

For all $f \in L^{1}(\mathfrak{G})$ define

$$
f^{\sharp}=\int_{\mathfrak{a}} f^{a^{\prime}} \psi\left(a^{\prime}\right) d a^{\prime} .
$$

As

$$
{ }^{a} \pi\left(f^{\prime \prime}\right)=\int_{\mathfrak{a}} a^{\prime \prime} \pi(f) \psi\left(a^{\prime \prime} a^{-1}\right) \delta\left(a, a^{\prime \prime}\right) d a^{\prime \prime},
$$

where $\delta\left(a, a^{\prime \prime}\right)$ stands for the Jacobian of the change of variables $a^{\prime \prime}=a^{\prime} a$, the kernel of the operator ${ }^{a} \pi\left(f^{\sharp}\right)$ is given by

$$
\widetilde{F}^{\sharp}(a ; . ; .)=\int_{\mathfrak{A}} \tilde{F}\left(a^{\prime \prime} ; \cdot ; \cdot\right) \psi\left(a^{\prime \prime} a^{-1}\right) \delta\left(a, a^{\prime \prime}\right) d a^{\prime \prime},
$$


where $\tilde{F}(a ; . ;$.$) is the kernel of the operator { }^{a} \pi(f), a \in \mathfrak{A}$. Put

$$
\left.\tilde{F}^{\sharp}\right|_{\mathfrak{B}}(a ; . ; .)=F^{\sharp}(a ; ; ; .)
$$

for the kernel of the operator ${ }^{a} \pi\left(f^{\sharp}\right)$ if we restrict ourselves to $a \in \mathfrak{V}$. If the support of $F$ in $a$ is contained in the compact set $K$ of $\mathfrak{V}$, then the support of $F^{\sharp}$ is contained in

$$
K^{\sharp}=\left(K_{0}^{-1} \cdot K \cdot \mathfrak{A}_{\pi}\right) \cap \mathfrak{V}=p\left(K_{0}^{-1} \cdot K\right),
$$

where $p$ is the projection of $\mathfrak{A}$ onto $\mathfrak{V}$. Since $p$ is continuous, $K^{\sharp}$ is a compact subset of $\mathfrak{V}$.

\section{RELATIONSHIP BETWEEN $\operatorname{Ker} \Omega$ AND $\operatorname{Ker} \Omega \cap \mathcal{S}(\mathfrak{G})$}

In order to study the relationship between $\operatorname{Ker} \Omega$ and $\operatorname{Ker} \Omega \cap \mathcal{S}(\mathfrak{B})$ we have to work with the kernels of the operators ${ }^{a} \pi(f)$, rather than with the functions $f$ themselves. The functions whose generalised Fourier transforms ${ }^{a} \pi(f)$ have compact support play an essential role in the proof. If the main result is rather complicated for general orbits (Proposition 4.1), it is very elegant for closed orbits (Theorem 4.2).

Definition: Let $K$ be a fixed compact subset of $\mathfrak{V}$ (identified with $\mathfrak{A} / \mathfrak{A}_{\pi}$ ). We define

$$
\mathcal{I}_{K}=\left\{f \in \mathcal{S}(\mathfrak{G}) \mid \overline{\left\{a \in \mathfrak{A} /\left.\mathfrak{A}_{\pi}\right|^{a} \pi(f) \neq 0\right\}} \subset K\right\}
$$

and

$$
\mathcal{I}=\left\{f \in \mathcal{S}(\mathfrak{G}) \mid \overline{\left\{a \in \mathfrak{A} /\left.\mathfrak{A}_{\pi}\right|^{a} \pi(f) \neq 0\right\}} \text { is compact }\right\} .
$$

Hence $\mathcal{I}=\bigcup_{K} \mathcal{I}_{K}$. Then $\mathcal{I}$ is an $\mathfrak{A}$-invariant ideal of $\mathcal{S}(\mathfrak{G})$. Obviously $\mathcal{I} \not \subset \operatorname{Ker} \pi$ by Theorem 3.1.

Proposition 4.1. With the notations of the previous definition

$$
\overline{\mathcal{I}}^{L^{1}(\mathfrak{B})} * \overline{\mathcal{I}}^{L^{1}(\mathfrak{B})} * \operatorname{Ker} \Omega * \overline{\mathcal{I}}^{L^{1}(\mathfrak{B})} * \overline{\mathcal{I}}^{L^{1}(\mathfrak{B})} \subset \overline{\operatorname{Ker} \Omega \cap \mathcal{S}(\mathfrak{B})}^{L^{1}(\mathcal{B})} .
$$

Proof: By the Hahn-Banach theorem it is enough to show that for $\varphi \in L^{\infty}(\mathfrak{G})$ such that $\langle\varphi, \operatorname{Ker} \Omega \cap \mathcal{S}(\mathfrak{G})\rangle=0$ we have

$$
\left\langle\varphi, \mathcal{I}_{K} * \mathcal{I}_{K} * \operatorname{Ker} \Omega * \mathcal{I}_{K} * \mathcal{I}_{K}\right\rangle=0 .
$$

Let $g_{1}, g_{2}, g_{3}, g_{4} \in \mathcal{I}_{K}$ and $f \in \mathcal{S}(\mathfrak{B})$. We write $G_{1}, G_{2}, G_{3}, G_{4}, F$ for the kernels of the operators ${ }^{a} \pi\left(g_{1}\right),{ }^{a} \pi\left(g_{2}\right),{ }^{a} \pi\left(g_{3}\right),{ }^{a} \pi\left(g_{4}\right),{ }^{a} \pi(f)$ if $a \in \mathfrak{V}$ and $\widetilde{G}_{1}, \widetilde{G}_{2}, \widetilde{G}_{3}, \widetilde{G}_{4}, \widetilde{F}$ for the kernels of the same operators if $a \in \mathfrak{A}$. We define a product of two such kernels by

$$
F \circ F_{1}(a, x, y)=\int_{\mathbf{R}^{k}} F\left(a, x, x_{1}\right) F_{1}\left(a, x_{1}, y\right) d x_{1}
$$


for all $F, F_{1} \in \mathcal{E S}\left(\mathfrak{V} ; \mathbb{R}^{k} \times \mathbb{R}^{k}\right)$. The product $\widetilde{F} \circ \widetilde{F}_{1}$ is defined similarly. According to this definition the kernel of ${ }^{a} \pi\left(g_{1} * g_{2} * f * g_{3} * g_{4}\right)$ is then given by $G_{1} \circ G_{2} \circ F \circ G_{3} \circ$ $G_{4}(a ; . ;$.$) if a \in \mathfrak{V}$ and by $\widetilde{G}_{1} \circ \widetilde{G}_{2} \circ \widetilde{F} \circ \widetilde{G}_{3} \circ \widetilde{G}_{4}(a ; ; ;)$ if $a \in \mathfrak{A}$. Hence

$$
\operatorname{supp}\left(G_{1} \circ G_{2} \circ F \circ G_{3} \circ G_{4}\right) \subset K \times \mathbb{R}^{k} \times \mathbb{R}^{k} .
$$

Let $\psi, K_{0}, K, K^{\sharp}$ be as in Section 3 and let us apply the construction of this section to the function $g_{1} * g_{2} * f * g_{3} * g_{4}$. The kernel function of ${ }^{a} \pi\left(\left(g_{1} * g_{2} * f * g_{3} * g_{4}\right)^{\sharp}\right)$ is given by

$$
\begin{aligned}
\int_{\mathfrak{A}} \widetilde{G}_{1} \circ \tilde{G}_{2} \circ \tilde{F} \circ \widetilde{G}_{3} \circ \widetilde{G}_{4}\left(a^{\prime \prime} ; . ; .\right) \psi\left(a^{\prime \prime} a^{-1}\right) \delta\left(a, a^{\prime \prime}\right) d a^{\prime \prime} & \\
= & \left(\widetilde{G}_{1} \circ \widetilde{G}_{2} \circ \widetilde{F} \circ \widetilde{G}_{3} \circ \widetilde{G}_{4}\right)^{\sharp}(a ; ; ; .)
\end{aligned}
$$

and the restriction of this kernel function to $\mathfrak{V}$ is an element of $\mathcal{E S}\left(\mathfrak{V} ; \mathbb{R}^{k} \times \mathbb{R}^{k}\right)$ whose support in $a$ is contained in the fixed compact set $K^{\sharp}$ of $\mathfrak{V}$, for every $f \in \mathcal{S}(\mathfrak{G})$. It is an element of the space $\mathcal{S}\left(K^{\sharp} ; \mathbb{R}^{k} \times \mathbb{R}^{k}\right)=\mathcal{D}\left(K^{\sharp} ; \mathcal{S}\left(\mathbb{R}^{k} \times \mathbb{R}^{k}\right)\right)$.

Next we define a continuous linear form $\mu$ on $\mathcal{E S}\left(\mathfrak{V} ; \mathbb{R}^{k} \times \mathbb{R}^{k}\right)$ in the following way: let $F \in \mathcal{E S}\left(\mathfrak{V} ; \mathbb{R}^{k} \times \mathbb{R}^{k}\right)$ and $f \in \mathcal{S}(\mathfrak{G})$ be such that the kernel of ${ }^{a} \pi(f)$ is given by $F(a ; . ;$.$) for every a \in \mathfrak{V}$. We define $\mu$ by $\langle\mu, F\rangle=\langle\varphi, f\rangle$. The linear form $\mu$ is well defined and continuous by Theorem 3.1 and by the fact that $\langle\varphi, \operatorname{Ker} \Omega \cap \mathcal{S}(\mathfrak{G})\rangle=0$. By $\left[23\right.$, p.239], the restriction of this linear form to the space $\mathcal{S}\left(K^{\sharp} ; \mathbb{R}^{k} \times \mathbb{R}^{k}\right)$ is of the form

$$
\langle\mu, F\rangle=\sum_{|\alpha|+|\beta|+|\gamma| \leqslant N} \int_{K^{\sharp} \times \mathbb{R}^{k} \times \mathbb{R}^{k}} \tau_{\alpha, \beta, \gamma}(a, x, y)\left(\frac{\partial}{\partial a}\right)^{\alpha}\left(\frac{\partial}{\partial x}\right)^{\beta}\left(\frac{\partial}{\partial y}\right)^{\gamma} F(a ; x ; y) d a d x d y
$$

where the $\tau_{\alpha, \beta, \gamma}$ 's are continuous functions of moderate growth and where $N \in \mathbb{N}$.

We may then define a new continuous linear form $\varphi^{\sharp}$ on $L^{1}(\mathfrak{G})$ by

$$
\left\langle\varphi^{\sharp}, f\right\rangle=\left\langle\varphi,\left(g_{1} * g_{2} * f * g_{3} * g_{4}\right)^{\sharp}\right\rangle \text {. }
$$

Let us show that

$$
\left|\left\langle\varphi^{\sharp}, f\right\rangle\right| \leqslant C \cdot \sup _{a \in K_{1}}\left\|^{a} \pi(f)\right\|_{o p} \leqslant\|f\|_{1}
$$

for a certain compact set $K_{1}$ of $\mathfrak{A}$ and for all $f \in \mathcal{S}(\mathfrak{B})$. In fact, for every $f \in \mathcal{S}(\mathfrak{B})$, we have

$$
\begin{aligned}
\left\langle\varphi^{\sharp}, f\right\rangle= & \left\langle\mu,\left.\left(\widetilde{G}_{1} \circ \widetilde{G}_{2} \circ \widetilde{F} \circ \widetilde{G}_{3} \circ \widetilde{G}_{4}\right)^{\sharp}\right|_{\mathfrak{V}}\right\rangle \\
= & \sum_{|\alpha|+|\beta|+|\gamma| \leqslant N} \int_{K^{\sharp} \times \mathbf{R}^{k} \times \mathbb{R}^{k}} \tau_{\alpha, \beta, \gamma}(a, x, y) \\
& \quad\left(\frac{\partial}{\partial a}\right)^{\alpha}\left(\frac{\partial}{\partial x}\right)^{\beta}\left(\frac{\partial}{\partial y}\right)^{\gamma}\left(\widetilde{G}_{1} \circ \widetilde{G}_{2} \circ \widetilde{F} \circ \widetilde{G}_{3} \circ \widetilde{G}_{4}\right)^{\sharp}(a, x, y) d a d x d y
\end{aligned}
$$


with

$$
\begin{aligned}
&\left(\widetilde{G}_{1} \circ \widetilde{G}_{2} \circ \widetilde{F} \circ \widetilde{G}_{3} \circ \widetilde{G}_{4}\right)^{\sharp}(a, x, y) \\
&=\int_{K_{1}} \int_{\mathbb{R}^{k} \times \mathbb{R}^{k}} \tilde{G}_{1}\left(a^{\prime \prime}, x, x^{\prime}\right) \widetilde{G}_{2} \circ \widetilde{F} \circ \widetilde{G}_{3}\left(a^{\prime \prime}, x^{\prime}, y^{\prime}\right) \widetilde{G}_{4}\left(a^{\prime \prime}, y^{\prime}, y\right) \\
& \psi\left(a^{\prime \prime} a^{-1}\right) \delta\left(a, a^{\prime \prime}\right) d x^{\prime} d y^{\prime} d a^{\prime \prime} .
\end{aligned}
$$

In fact, the integral on $a^{\prime \prime}$ may be limited to the compact subset $K_{1}=K_{0} \cdot K^{\sharp}$ of $\mathfrak{A}$, as the support of $\psi$ is the compact set $K_{0}$ and as $a$ takes its values in the compact set $K^{\sharp}$ of $\mathfrak{V} \subset \mathfrak{A}$. Hence

$$
\begin{aligned}
&\left(\frac{\partial}{\partial a}\right)^{\alpha}\left(\frac{\partial}{\partial x}\right)^{\beta}\left(\frac{\partial}{\partial y}\right)^{\gamma}\left(\widetilde{G}_{1} \circ \widetilde{G}_{2} \circ \tilde{F} \circ \widetilde{G}_{3} \circ \widetilde{G}_{4}\right)^{\sharp}(a, x, y) \\
&=\int_{K_{1}}\left\{\int_{\mathbb{R}^{k} \times \mathbb{R}^{k}}\left[\left(\frac{\partial}{\partial x}\right)^{\beta} \tilde{G}_{1}\left(a^{\prime \prime}, x, x^{\prime}\right)\right] \widetilde{G}_{2} \circ \widetilde{F} \circ \widetilde{G}_{3}\left(a^{\prime \prime}, x^{\prime}, y^{\prime}\right)\right. \\
&\left.\quad\left[\left(\frac{\partial}{\partial y}\right)^{\gamma} \widetilde{G}_{4}\left(a^{\prime \prime}, y^{\prime}, y\right)\right] d x^{\prime} d y^{\prime}\right\}\left[\left(\frac{\partial}{\partial a}\right)^{\alpha} \psi\left(a^{\prime \prime} a^{-1}\right) \delta\left(a, a^{\prime \prime}\right)\right] d a^{\prime \prime} .
\end{aligned}
$$

Let us write

$$
\begin{aligned}
\widetilde{G}_{\beta, 1}^{\prime}\left(a^{\prime \prime}, x, x^{\prime}\right) & =\left(\frac{\partial}{\partial x}\right)^{\beta} \widetilde{G}_{1}\left(a^{\prime \prime}, x, x^{\prime}\right), \\
\widetilde{G}_{\gamma, 4}^{\prime}\left(a^{\prime \prime}, y^{\prime}, y\right) & =\left(\frac{\partial}{\partial y}\right)^{\gamma} \widetilde{G}_{4}\left(a^{\prime \prime}, y^{\prime}, y\right), \\
\psi^{\prime}\left(a, a^{\prime \prime}\right) & =\left(\frac{\partial}{\partial a}\right)^{\alpha} \psi\left(a^{\prime \prime} a^{-1}\right) \delta\left(a, a^{\prime \prime}\right) .
\end{aligned}
$$

The Cauchy-Schwarz inequality then gives us the following estimate:

$$
\begin{aligned}
&\left|\left\langle\varphi^{\sharp}, f\right\rangle\right| \leqslant\left\|\tilde{G}_{2} \circ \tilde{F} \circ \tilde{G}_{3}\right\|_{L^{2}\left(K_{1} \times \mathbb{R}^{k} \times \mathbb{R}^{k}\right)} \\
& \cdot \sum_{|\alpha|+|\beta|+|\gamma| \leqslant N}\left\{\int _ { K _ { 1 } \times \mathbb { R } ^ { k } \times \mathbb { R } ^ { k } } \left[\int_{K^{\sharp} \times \mathbf{R}^{k} \times \mathbf{R}^{k}} \mid \tau_{\alpha, \beta, \gamma}(a, x, y,) \psi^{\prime}\left(a, a^{\prime \prime}\right)\right.\right. \\
&\left.\left.\tilde{G}_{\beta, 1}^{\prime}\left(a^{\prime \prime}, x, x^{\prime}\right) \widetilde{G}_{\gamma, 4}^{\prime}\left(a^{\prime \prime}, y^{\prime}, y\right) \mid d a d x d y\right]^{2} d a^{\prime \prime} d x^{\prime} d y^{\prime}\right\}^{1 / 2} .
\end{aligned}
$$

By the continuity of the mapping

$$
\begin{aligned}
\left(a, a^{\prime \prime}\right) \mapsto\left|\psi^{\prime}\left(a, a^{\prime \prime}\right)\right| \cdot \int_{\mathbf{R}^{k} \times \mathbf{R}^{k}} \int_{\mathbf{R}^{k} \times \mathbf{R}^{k}} \mid \tau_{\alpha, \beta, \gamma}(a, x, y) & \\
& \widetilde{G}_{\beta, 1}^{\prime}\left(a^{\prime \prime}, x, x^{\prime}\right) \widetilde{G}_{\gamma, 4}^{\prime}\left(a^{\prime \prime}, y^{\prime}, y\right) \mid d x d y d x^{\prime} d y^{\prime},
\end{aligned}
$$


this mapping is bounded on $K^{\sharp} \times K_{1}$ and one shows an inequality of the type

$$
\begin{aligned}
\left|\left\langle\varphi^{\sharp}, f\right\rangle\right| & \leqslant C \cdot\left\|\widetilde{G}_{2} \circ \widetilde{F} \circ \widetilde{G}_{3}\right\|_{L^{2}\left(K_{1} \times \mathbf{R}^{k} \times \mathbf{R}^{k}\right)} \\
& \leqslant C \cdot \operatorname{meas} K_{1} \cdot \sup _{a \in K_{1}}\left\|\widetilde{G}_{2} \circ \widetilde{F} \circ \widetilde{G}_{3}(a, ., .)\right\|_{L^{2}\left(\mathbf{R}^{k} \times \mathbf{R}^{k}\right)} \\
& =C \cdot \operatorname{meas} K_{1} \cdot \sup _{a \in K_{1}}\left\|^{a} \pi\left(g_{2}\right)^{a} \pi(f)^{a} \pi\left(g_{3}\right)\right\|_{\mathrm{HS}},
\end{aligned}
$$

as the Hilbert-Schmidt norm $\|\cdot\|_{\mathrm{HS}}$ of an operator coincides with the $L^{2}$-norm of its kernel. Finally,

$$
\begin{aligned}
\left|\left\langle\varphi^{\sharp}, f\right\rangle\right| & \leqslant C_{1} \cdot \sup _{a \in K_{1}}\left[\left\|^{a} \pi\left(g_{2}\right)\right\|_{H S} \cdot\left\|^{a} \pi\left(g_{3}\right)\right\|_{H S}\right] \cdot \sup _{a \in K_{1}}\left\|^{a} \pi(f)\right\|_{\text {op }} \\
& \leqslant C_{2} \cdot \sup _{a \in K_{1}}\left\|^{a} \pi(f)\right\|_{\text {op }} \\
& \leqslant C_{2} \cdot\|f\|_{1},
\end{aligned}
$$

for every $f \in \mathcal{S}(\mathfrak{G})$ and some new constants $C_{1}, C_{2}$. As $\mathcal{S}(\mathfrak{G})$ is dense in $L^{1}(\mathfrak{G})$ and as the linear form $\varphi^{\sharp}$ is continuous on $L^{1}(\mathfrak{G})$, the inequality

$$
\left|\left\langle\varphi^{\sharp}, f\right\rangle\right| \leqslant C_{2} \cdot \sup _{a \in K_{1}}\left\|^{a} \pi(f)\right\|_{o p} \leqslant C_{2} \cdot\|f\|_{1}
$$

remains true for every $f \in L^{1}(\mathfrak{G})$. In particular, if $f \in \operatorname{Ker} \Omega,{ }^{a} \pi(f)=0$ for all $a$ and

$$
\begin{aligned}
0 & =\left\langle\varphi^{\sharp}, f\right\rangle \\
& =\left\langle\varphi,\left(g_{1} * g_{2} * f * g_{3} * g_{4}\right)^{\sharp}\right\rangle \\
& =\int_{K_{0}} \int_{\mathfrak{G}} \varphi(x)\left(g_{1} * g_{2} * f * g_{3} * g_{4}\right)^{a}(x) \psi(a) d x d a .
\end{aligned}
$$

Let us now choose the functions $\psi$ such that their supports form a basis of neighbourhoods of the origin. We then see that

$$
\int_{\mathfrak{B}} \varphi(x)\left(g_{1} * g_{2} * f * g_{3} * g_{4}\right)(x) d x=0
$$

This completes the proof.

THEOREM 4.2 . If $\Omega$ is a closed orbit in $\mathfrak{g}^{*}$, then

$$
\overline{\operatorname{Ker} \Omega \cap \mathcal{S}(\mathfrak{G})}^{L^{1}(\mathfrak{B})}=\operatorname{Ker} \Omega \text {. }
$$

PROOF: If $\left(g_{\nu}\right)$ is the approximate identity of Proposition 2.2, then $g_{\nu} \in \mathcal{I}$ and, for all $f \in \operatorname{Ker} \Omega$,

$$
g_{\nu} * g_{\nu} * f * g_{\nu} * g_{\nu} \in \mathcal{I} * \mathcal{I} * \operatorname{Ker} \Omega * \mathcal{I} * \mathcal{I} \subset \overline{\operatorname{Ker} \Omega \cap \mathcal{S}(\mathfrak{B})}^{L}(\mathfrak{B})
$$

By taking the limit in $\nu$ we complete the proof. 
REMARKS.

(a) This relationship will allow us to prove certain results first for $\mathcal{S}(\mathfrak{G})$ and to generalise them afterwards to $L^{1}(\mathfrak{G})$.

(b) In [12, 2.1 Proposition] Ludwig shows a similar result for the kernel of an irreducible unitary representation of a connected, simply connected nilpotent Lie group.

(c) Nothing seems to be known about the relationship between $\operatorname{Ker} \Omega$ and $\operatorname{Ker} \Omega \cap \mathcal{S}(\mathfrak{G})$, if the orbit $\Omega$ is not closed.

\section{Applications}

The main consequences of the relationship between $\operatorname{Ker} \Omega \cap \mathcal{S}(\mathfrak{G})$ and $\operatorname{Ker} \Omega$ are a generalised spectral synthesis result for Ker $\Omega$ (Theorem 5.1) and the characterisation of the $\mathfrak{A}$-invariant prime ideals of $L^{1}(\mathfrak{B})$ (Theorem 5.3). But first we have to recall some questions of topology and some results on minimal ideals.

Let $\mathfrak{G}$ be a connected, simply connected nilpotent Lie group. Then the topological spaces $\widehat{\mathfrak{G}}, \mathfrak{g}^{*} / \operatorname{Ad}^{*} \mathfrak{G}, \operatorname{Prim}_{*} L^{1}(\mathfrak{B})$ and $\operatorname{Prim} \mathcal{S}(\mathfrak{G})$ are homeomorphic by $[\mathbf{2}, \mathbf{3}, \mathbf{9}, 15]$. In particular, the homeomorphism between $\widehat{\mathscr{G}}$ and $\operatorname{Prim}_{*} L^{1}(\mathfrak{G})$ is given by

$$
\begin{aligned}
\widehat{\mathfrak{G}} & \rightarrow \operatorname{Prim}_{*} L^{1}(\mathfrak{G}) \\
\pi & \mapsto \operatorname{Ker} \pi
\end{aligned}
$$

as the group $\mathfrak{G}$ is *-regular [2, Satz 2]. Hence, if $C \subset \widehat{\mathfrak{G}}$,

$$
\pi_{l} \in \bar{C} \Longleftrightarrow \operatorname{Ker} C \subset \operatorname{Ker} \pi_{l}
$$

and for an arbitrary orbit $\Omega$,

$$
\operatorname{Ker} \Omega \subset \operatorname{Ker} \pi_{l} \Longleftrightarrow l \in \bar{\Omega}
$$

(see [18]). This implies that for any two orbits $\Omega_{1}$ and $\Omega_{2}$ one has

$$
\operatorname{Ker} \Omega_{1}=\operatorname{Ker} \Omega_{2} \Longleftrightarrow \overline{\Omega_{1}}=\overline{\Omega_{2}} \text {. }
$$

Minimal ideals in $\mathcal{S}(\mathfrak{G})$ and $L^{1}(\mathfrak{G})$. Let $\Omega$ be a closed orbit in $\mathfrak{g}^{*}$. Hence $\Omega / \operatorname{Ad}^{*} \mathfrak{G}$ is a closed subset of $\widehat{\mathfrak{G}} \equiv \mathfrak{g}^{*} / \operatorname{Ad}^{*} \mathfrak{G} \equiv \operatorname{Prim}_{*} L^{1}(\mathfrak{B}) \equiv \operatorname{Prim} \mathcal{S}(\mathfrak{G})$. There exists a minimal ideal $j(\Omega)$ in $\mathcal{S}(\mathfrak{G})$ such that its hull satisfies $h(j(\Omega))=\Omega$ and such that $j(\Omega)$ is contained in every ideal $I$ of $\mathcal{S}(\mathfrak{G})$ satisfying $h(I) \subset \Omega$. In [15, 3.2] and [17] it is shown that $j(\Omega)$ is generated by all the functions of the form $\varphi\{f\}$ with $f=f^{*} \in \operatorname{Ker} \Omega \cap \mathcal{S}(\mathfrak{G})$ and where $\varphi \in C^{\infty}(\mathbb{R})$ is identically zero in a neighbourhood of 0 . The functions $\varphi\{f\}$ are given by the functional calculus of Dixmier [4]. Therefore the closure of $j(\Omega)$ in $L^{1}(\mathfrak{B})$, denoted by $\widetilde{\jmath}(\Omega)$, is the minimal closed ideal in $L^{1}(\mathfrak{B})$ whose bull $h(\tilde{\jmath}(\Omega))$ is equal to $\Omega$. 
REMARK. In [15, 3.5] Ludwig shows the following result for $\mathcal{S}(\mathfrak{G})$. Let $\Omega$ be a closed orbit in $\mathfrak{g}^{*}$. There exists $M \in \mathbb{N}$ such that

$$
(\operatorname{Ker} \Omega \cap \mathcal{S}(\mathfrak{G}))^{M} \subset \overline{j(\Omega)}^{L^{1}(\mathbb{B})}=\tilde{\jmath}(\Omega) .
$$

This result can now be transferred to the algebra $L^{1}(\mathfrak{B})$.

ThEOREM 5.1. Let $\Omega$ be a closed orbit in $\mathfrak{g}^{*}$. Then there exists $M \in \mathbb{N}$ such that

$$
(\operatorname{Ker} \Omega / \widetilde{\jmath}(\Omega))^{M}=\{0\} .
$$

This means that the algebra $\operatorname{Ker} \Omega / \widetilde{\jmath}(\Omega)$ is nilpotent.

PROOF: By the preceding remark and Theorem 4.2. REMARKS.

(a) In [16, Theorem 7] Ludwig shows a similar result for $\operatorname{Ker} \pi$ where $\pi$ is a unitary irreducible representation of a nilpotent Lie group.

(b) Poguntke proves similar results for other actions: for a nilpotent action on a nilpotent Lie group [20, Theorem 5] and for the action of a semidirect product of a compact Abelian group with a nilpotent Lie group on a nilpotent Lie group [21, Satz 7].

Definition: A proper $\mathfrak{A}$-invariant ideal $I$ of $L^{1}(\mathfrak{G})(\mathcal{S}(\mathfrak{B}))$ is said to be $\mathfrak{A}$-prime whenever for all $\mathfrak{A}$-invariant ideals $I_{1}$ and $I_{2}$ of $L^{1}(\mathfrak{G})$, (respectively, $\mathcal{S}(\mathfrak{G})$ ),

$$
I_{1} * I_{2} \subset I \Rightarrow I_{1} \subset I \text { or } I_{2} \subset I
$$

Lemma 5.2. If we identify the spaces $\operatorname{Prim} \mathcal{S}(\mathfrak{G})$ and $\operatorname{Prim}_{*} L^{1}(\mathfrak{G})$, the hull of every closed ideal $I$ of $L^{1}(\mathfrak{G})$ satisfies

$$
h(I)=h(I \cap \mathcal{S}(\mathfrak{G}))
$$

Proof: The identification between $\operatorname{Prim}_{*} L^{1}(\mathfrak{G})$ and $\operatorname{Prim} \mathcal{S}(\mathfrak{G})$ is given by $\operatorname{Ker} \pi \mapsto \operatorname{Ker} \pi \cap S(\mathfrak{G}) \quad([15,3.1]$ and [2, Satz 2]). This implies the inclusion $h(I) \subset h(I \cap \mathcal{S}(\mathfrak{G}))$. On the other hand, the relation

$$
\overline{j(h(I))}^{L^{1}(\mathfrak{B})}=\tilde{\jmath}(h(I)) \subset I
$$

shows that $j(h(I)) \subset I \cap \mathcal{S}(\mathfrak{G})$, which in its turn implies that $h(I \cap \mathcal{S}(\mathfrak{G})) \subset$ $h(j(h(I)))=h(I)$. 
THEOREM 5.3. The proper closed $\mathfrak{A}$-prime ideals of $L^{1}(\mathfrak{G})$ coincide with the kernels Ker $\Omega$ of the orbits $\Omega$ of $\mathfrak{g}^{*}$. Moreover the map

$$
\Omega \mapsto \operatorname{Ker} \Omega
$$

is a bijection between the set of orbits of $\mathfrak{g}^{*}$ and the set of proper closed $\mathfrak{A}$-prime ideals of $L^{1}(\mathfrak{G})$.

Proof: It is obvious that the ideals $\operatorname{Ker} \Omega$ are $\mathfrak{A}$-prime. Conversely, suppose that $I$ is a proper closed $\mathfrak{A}$-prime ideal of $L^{1}(\mathfrak{G})$. Then $I_{S}=I \cap \mathcal{S}(\mathfrak{G})$ is a proper $\mathfrak{A}$-invariant ideal of $\mathcal{S}(\mathfrak{G})$ which is closed in the continuous $\|\cdot\|_{1}$ norm. Moreover, $I_{S}$ is $\mathfrak{A}$-prime. In fact, if $\mathcal{I}_{1}$ and $\mathcal{I}_{2}$ are $\mathfrak{A}$-invariant ideals of $\mathcal{S}(\mathfrak{G})$ such that $\mathcal{I}_{1} * \mathcal{I}_{2} \subset I_{S}$, then

$$
{\overline{\mathcal{I}_{1}}}^{L^{1}(\mathcal{B})} *{\overline{\mathcal{I}_{2}}}^{L^{1}(\mathfrak{B})} \subset{\overline{I_{S}}}^{L^{1}(\mathfrak{B})} \subset I .
$$

As $I$ is $\mathfrak{A}$-prime, ${\overline{\mathcal{I}_{1}}}^{L^{1}(\mathfrak{B})} \subset I$ or ${\overline{\mathcal{I}_{2}}}^{L^{1}(\mathcal{B})} \subset I$. Hence $\mathcal{I}_{1} \subset I_{S}$ or $\mathcal{I}_{2} \subset I_{S}$. By [18, 9.8] there is an orbit $\Omega$ such that $I_{S}=\operatorname{Ker} \Omega \cap \mathcal{S}(\mathfrak{G})$. Hence, by Lemma 5.2,

$$
h(I)=h\left(I_{S}\right)=h(\operatorname{Ker} \Omega \cap \mathcal{S}(\mathfrak{G}))=h(\operatorname{Ker} \Omega)=\bar{\Omega}
$$

and $I \subset \operatorname{Ker} \Omega$. With the notation of Proposition 4.1 we have

$$
\begin{aligned}
\overline{\mathcal{I}}^{L^{1}(\mathfrak{B})} * \overline{\mathcal{I}}^{L^{1}(\mathfrak{B})} * \operatorname{Ker} \Omega * \overline{\mathcal{I}}^{L^{1}(\mathfrak{B})} * \overline{\mathcal{I}}^{L^{1}(\mathcal{B})} & \subset \overline{\operatorname{Ker} \Omega \cap \mathcal{S}(\mathfrak{B})}^{L^{1}(\mathfrak{B})} \\
& =\overline{I \cap \mathcal{S}(\mathcal{B})}^{L^{1}(\mathfrak{B})} \\
& \subset I .
\end{aligned}
$$

As $\mathcal{I} \not \subset \operatorname{Ker} \Omega$ and $I \subset \operatorname{Ker} \Omega, \mathcal{I} \not \subset I$. Hence $\operatorname{Ker} \Omega \subset I$ and $I=\operatorname{Ker} \Omega$.

To finish the proof, we just have to show the injectivity of the mapping $\Omega \mapsto \operatorname{Ker} \Omega$. Suppose that $\Omega_{1}$ and $\Omega_{2}$ are two distinct orbits such that $\operatorname{Ker} \Omega_{1}=\operatorname{Ker} \Omega_{2}$. Hence $\bar{\Omega}_{1}=h\left(\operatorname{Ker} \Omega_{1}\right)=h\left(\operatorname{Ker} \Omega_{2}\right)=\bar{\Omega}_{2}, \Omega_{1} \subset \bar{\Omega}_{2} \backslash \Omega_{2}$ and $\Omega_{2} \subset \bar{\Omega}_{1} \backslash \Omega_{1}$. As every orbit is open in its closure, $\bar{\Omega}_{1} \backslash \Omega_{1}$ and $\bar{\Omega}_{2} \backslash \Omega_{2}$ are closed and

$$
\Omega_{1} \subset \bar{\Omega}_{1} \subset \bar{\Omega}_{2} \backslash \Omega_{2} \subset \bar{\Omega}_{2} \subset \bar{\Omega}_{1} \backslash \Omega_{1}
$$

This contradiction shows that $\Omega_{1}=\Omega_{2}$.

REMARK. In $[14,10$, Theorem] Ludwig shows the corresponding result without exterior action: the proper closed prime ideals of $L^{1}(\mathfrak{G})$ coincide with the kernels $\operatorname{Ker} \pi$ where $\pi \in \widehat{\mathfrak{G}}$. 


\section{REFERENCES}

[1] P. Bernat, N. Conze, M. Duflo, M. Lévy-Nahas, M. Rais, P. Renouard and M. Vergne, Représentations des groupes de Lie résolubles (Dunod, Paris, 1972).

[2] J. Boidol, H. Leptin, J. Schürman and D. Vahle, 'Räume primitiver Ideale von Gruppenalgebren', Math. Ann. 236 (1978), 1-13.

[3] I. Brown, 'Dual topology of a nilpotent Lie group', Ann. Sci. École Norm. Sup. 6 (1973), 407-411.

[4] J. Dixmier, 'Opérateurs de rang fini dans les représentations unitaires', Inst. Hautes Études Sci. Publ. Math. 6 (1960), 305-317.

[5] J. Dixmier, Les $C^{*}$-algèbres et leurs représentations (Gauthier-Villars, Paris, 1969).

[6] G. Hochschild, The structure of Lie groups (Holden-Day Inc., San Francisco, London, Amsterdam, 1965).

[7] R. Howe, 'On a connection between nilpotent groups and oscillatory integrals associated to singularities', Pacific J. Math. 73 (1977), 329-363.

[8] A. Hulanicki, 'A functional calculus for Rockland operators on nilpotent Lie groups', Stud. Math. 78 (1984), 253-266.

[9] A.A. Kirillov, 'Unitary representations of nilpotent Lie groups', Uspekhi Mat. Nauk. 17 (1962), 53-104.

[10] H. Leptin, 'Ideal theory in group algebras of locally compact groups', Invent. Math. 31 (1976), 259-278.

[11] H. Leptin and J. Ludwig, Unitary representation theory of exponential Lie groups, De Gruyter Expositions in Mathematics 18 (De Gruyter, Berlin, New York, 1994).

[12] J. Ludwig, 'On the spectral synthesis problem for points in the dual of a nilpotent Lie group', Ark. Mat. 21 (1983), 127-144.

[13] J. Ludwig, 'Irreducible representations of exponential solvable Lie groups and operators with smooth kernels', J. Reine Angew. Math. 339 (1983), 1-26.

[14] J. Ludwig, 'On primary ideals in the group algebra of a nilpotent Lie group', Math. Ann. 262 (1983), 287-304.

[15] J. Ludwig, 'Minimal $C^{*}$-dense ideals and algebraically irreducible representations of the Schwartz-algebra of a nilpotent Lie group', in Harmonic analysis, Lecture Notes in Math. 1359 (Springer Verlag, Berlin, Heidelberg, New York, 1987), pp. 209-217.

[16] J. Ludwig and C. Molitor-Braun, 'Algèbre de Schwartz d'un groupe de Lie nilpotent', Centre Univ. Luxembourg, Travaux mathématiques VII (1995), 25-67.

[17] J. Ludwig, G. Rosenbaum and J. Samuel, 'The elements of bounded trace in the $C^{*}$-algebra of a nilpotent Lie group', Invent. Math. 83 (1986), 167-190.

[18] C. Molitor-Braun, Actions exponentielles et idéaux premiers, Thèse (Metz, 1996).

[19] C. Molitor-Braun, 'Actions exponentielles et noyaux d'opérateurs', Centre Univ. Luxembourg, Travaux mathématiques IX (1997), 23-101.

[20] D. Poguntked, 'Algebraically irreducible representations of $L^{1}$-algebras of exponential Lie groups', Duke Math. J. 50 (1983), 1077-1106. 
[21] D. Poguntke, 'Über das Synthese-problem für nilpotente Liesche Gruppen', Math. Ann. 269 (1984), 431-467.

[22] L. Pukanszky, 'On the unitary representations of exponential groups', J. Funct. Anal. 2 (1968), 73-113.

[23] L. Schwartz, Théorie des distributions (Hermann, Paris, 1973).

Département de Mathématiques

Université de Metz

Ile de Saulcy

F-57045 Metz cedex 1

France
Séminaire de Mathématique

Centre Universitaire de Luxembourg

162A Avenue de la Faïencerie

L-1511 Luxembourg

Luxembourg 